\title{
Phase II Trial of Carboplatin and Pemetrexed Plus Bevacizumab with Maintenance Bevacizumab as a First-line Treatment for Advanced Non-squamous Non-small Cell Lung Cancer in Elderly Patients
}

\author{
HIROAKI TAKEOKA $^{1}$, KAZUHIKO YAMADA $^{2}$, YOSHIKO NAITO ${ }^{2}$, NORIKAZU MATSUO ${ }^{2}$, \\ HIDENOBU ISHII ${ }^{2}$, TAKAAKI TOKITO ${ }^{2}$, KOICHI AZUMA ${ }^{2}$, MASAO ICHIKI $^{1}$ and TOMOAKI HOSHINO ${ }^{2}$ \\ ${ }^{1}$ Department of Respiratory Medicine, Clinical Research Institute, \\ National Hospital Organization, Kyushu Medical Center, Fukuoka, Japan; \\ ${ }^{2}$ Division of Respirology, Neurology, and Rheumatology, Department of Internal Medicine, \\ Kurume University School of Medicine, Kurume, Japan
}

\begin{abstract}
Background/Aim: The combination of platinumdoublet chemotherapy with bevacizumab has been established as a first-line treatment option in non-elderly patients with non-squamous (non-sq) non-small cell lung cancer (NSCLC). However, the safety and efficacy of this regimen have not yet been fully established in elderly patients. Patients and Methods: Chemo-naïve patients with non-sq NSCLC, aged $\geq 75$ years, having a good performance status (Eastern Cooperative Oncology Group performance status 0-1) and adequate organ function were considered eligible. Patients received carboplatin (area under the curve $=5 \mathrm{mg} / \mathrm{ml} / \mathrm{min})$, pemetrexed $\left(500 \mathrm{mg} / \mathrm{m}^{2}\right)$, and bevacizumab (15 mg/kg) every 3 weeks for up to 4 cycles, followed by maintenance bevacizumab. The primary endpoint was the objective response rate (ORR; target $=50 \%$, threshold $=30 \%$; Simon's two-stage design), and the secondary endpoints were safety, progression-free survival (PFS), and overall survival (OS). Results: Twelve patients were enrolled from June 2013 to July 2017. The study was closed because of slow patient accrual. The median patient age was 80 years. Eleven patients (92\%) completed 4 cycles of induction chemotherapy. Seven patients achieved a partial response (PR), yielding an ORR of 58\%. The median PFS was 8.4 [95\% confidence interval $(C I)=4.4-10.5]$ months,
\end{abstract}

Correspondence to: Kazuhiko Yamada, MD, Ph.D., Division of Respirology, Neurology, and Rheumatology, Department of Internal Medicine, Kurume University School of Medicine, 67 Asahi-machi, Kurume City, Fukuoka, 830-0011, Japan. Tel: +81 942317560, Fax: +81 942317703, e-mail: kayamada@med.kurume-u.ac.jp

Key Words: Carboplatin, pemetrexed, bevacizumab, elderly, NSCLC. and the median OS was 33.9 (95\%CI=13.2-43.3) months. Toxicities were generally mild and consistent with previous reports. There were no treatment-related deaths. Conclusion: A regimen comprising carboplatin and pemetrexed plus bevacizumab followed by maintenance bevacizumab is feasible and potentially efficacious in elderly patients with non-sq NSCLC.

Lung cancer is the most common type of cancer and leading cause of cancer-related deaths worldwide. Approximately $85 \%$ of lung cancer cases involve the non-small-cell type (NSCLC), and most patients have advanced-stage disease at the time of diagnosis (1). The frequency of elderly (aged $\geq 75$ years) lung cancer patients has increased significantly among the overall lung cancer population, consistent with trends in societal aging. In Japan, approximately half of all newly diagnosed cases of lung cancer involve patients aged $\geq 75$ years (2), and this tendency is likely to increase in future.

Currently, platinum-doublet chemotherapy is the standard treatment administered to patients with advanced NSCLC. However, elderly patients are more likely to present with impaired organ function and comorbidities, compared to younger patients, and are more likely to experience exacerbations of treatment-related toxicities and underlying comorbidities, that reduce the potential survival benefits of treatment. Therefore, elderly patients have been systematically excluded from clinical studies and accordingly data regarding the role of platinum-doublet chemotherapy in this population remain scarce.

Two prospective randomized controlled studies of elderly patients with NSCLC demonstrated that vinorelbine and docetaxel monotherapies improved both the quality of life (QOL) and survival (3, 4). Accordingly, such monotherapies are currently used as standard treatments for 
elderly patients with NSCLC. However, decisions regarding the treatment of elderly patients should not be made only in terms of chronological age. Retrospective analyses of age-specific subgroups in two phase III studies indicated that despite an increased incidence of toxicity, platinumdoublet chemotherapy may be feasible and efficacious in otherwise healthy elderly patients with a good performance Status (PS) and adequate organ function $(5,6)$. The IFCT0501 study of elderly (aged $\geq 70$ years) patients with NSCLC demonstrated the survival benefits of carboplatin plus weekly paclitaxel, compared with vinorelbine or gemcitabine monotherapy. However, treatment-related deaths were observed more frequently with platinumdoublet chemotherapy than with monotherapy (7). Therefore, more effective and less toxic chemotherapies are needed for elderly patients with NSCLC.

We previously reported a phase I dose-escalated study of carboplatin plus pemetrexed for elderly patients (aged $\geq 75$ years). In that study, the patients exhibited good tolerance of the toxicities associated with carboplatin plus pemetrexed therapy (8). Another dose-escalation study reported by a group in Japan yielded similar results (9).

The AVAiL and ECOG4599 studies demonstrated that a combination of platinum-doublet chemotherapy plus bevacizumab, a monoclonal antibody specific for vascular endothelial growth factor, significantly improved progressionfree survival (PFS) and overall survival (OS), compared with platinum-doublet chemotherapy alone $(10,11)$. Those studies demonstrated a trend toward promising responses and survival benefits when bevacizumab was added to a platinumdouble chemotherapy regimen administered to elderly patients with non-sq NSCLC $(12,13)$. However, the results of those clinical studies are not generalizable to all nonselected elderly patients with NSCLC. Therefore, we conducted a prospective study to evaluate the safety and tolerability of this combination chemotherapy in a population of elderly patients (age $\geq 75$ years) with non-sq NSCLC.

\section{Patients and Methods}

Study design. This was a multicenter single-arm phase II study of carboplatin and pemetrexed plus bevacizumab, followed by maintenance bevacizumab. The primary endpoint was the objective response rate (ORR), and the secondary endpoints were safety, PFS, and OS.

Eligibility. Patients aged $\geq 75$ years were required to have histologically or cytologically confirmed non-sq NSCLC for which they had not received systemic chemotherapy except tegafur-uracil for adjuvant therapy or therapy for pleurodesis and first-line therapy comprising of an epidermal growth factor receptor (EGFR) tyrosine kinase inhibitor (TKI) or anaplastic lymphoma kinase (ALK) TKI. Additional inclusion criteria included measurable disease according to the Response Evaluation Criteria in Solid Tumors (RECIST v.1.1), an Eastern Cooperative
Oncology Group Performance Status (PS) of 0 or 1, adequate organ function (e.g., hematologic, hepatic, and renal systems) within 1 week before study entry, and an expected survival of $>90$ days after enrollment. The exclusion criteria were a previous treatment history, pleural or pericardial effusion requiring drainage, metastatic brain tumor requiring treatment (e.g., radiotherapy or steroid therapy), a high risk of hemorrhage risk (e.g., requirement for anticoagulant and antiplatelet therapy), a history of active hemoptysis (volume $\geq 2.5 \mathrm{ml}$ ), tumor invasion to the great vessels and cavity lesions, clinically significant cardiovascular disease, uncontrolled hypertension (regardless of hypotensive drug use), and a judgment by the attending physician of inadequacy to participate safely in the study.

The study was conducted in accordance with the ethical standards established in the 1964 Declaration of Helsinki and its later amendments. The study was approved by the institutional review board of each study site, and all patients participating in the study provided written informed consent before enrollment. This study was registered with the University Hospital Medical Information Network (UMIN) in Japan (number UMIN 000011095).

Study treatment. Eligible patients were treated with up to 4 cycles of carboplatin at a dose calculated to produce an area under the curve of $5 \mathrm{mg} / \mathrm{ml} / \mathrm{min}$, pemetrexed at a dose of $500 \mathrm{mg} / \mathrm{m}^{2}$, and bevacizumab at a dose of $15 \mathrm{mg} / \mathrm{kg}$; all drugs were administered on day 1 of a 21-day cycle. Patients without disease progression during the induction phase were eligible to continue bevacizumab at a dose of $15 \mathrm{mg} / \mathrm{kg}$ administered on day 1 of a 21-day cycle until disease progression, death, intolerable toxicity, or consent withdrawal occurred. During the induction phase, all patients received folic acid and vitamin $B_{12}$ supplements until 3 weeks after the last dose of pemetrexed administration.

Efficacy and safety assessments. Following a baseline evaluation, the tumor status was evaluated every 6 weeks during the first 24 weeks and then every 12 weeks until study treatment termination. The ORR was assessed from computed tomography and magnetic resonance imaging findings in accordance with RECIST 1.0. Adverse events (AEs) were monitored every 1 or 2 weeks during study treatment and graded according to the National Cancer Institute (NCI)-Common Terminology Criteria for Adverse Events version 3.0.

Statistical analysis. The primary endpoint of this study was the ORR, which was estimated as the confirmed response [complete response + partial response $(\mathrm{CR}+\mathrm{PR})]$. The secondary endpoints included safety, PFS, and OS. Our previous study of elderly patients (8) reported a response rate of approximately $30 \%$ for carboplatin plus pemetrexed, and previous phase III studies of bevacizumab plus platinum-doublet chemotherapy reported response rates approximately $20 \%$ higher than those achieved with platinum-doublet chemotherapy alone $(10,11)$. Based on a Simon's 2-stage design (14), a sample size of 42 was estimated to accomplish a power of $\geq 80 \%$ with a 1 -sided alpha $=0.05$, with a threshold and expected value of $30 \%$ and $50 \%$, respectively. Survival estimations were calculated using the Kaplan-Meier method. PFS was defined from the time of registration to the time of the first documented disease progression or mortality. OS was defined from the time of registration to the time of mortality from any cause. 
Table I. Patient characteristics.

\begin{tabular}{lc}
\hline Number of patients & 12 \\
Gender & \\
$\quad$ Male/Female & $4 / 8$ \\
Median age, years (range) & $80(75-83)$ \\
Performance status & \\
$\quad 0 / 1$ & $8 / 4$ \\
Histology & \\
$\quad$ Ad & 12 \\
Stage & \\
IIIA/IIIB/IV/Relapse after surgery & $1 / 0 / 9 / 2$ \\
EGFR mutation status & \\
$\quad$ Exon 19/Exon 21 & $2 / 2$ \\
$\quad$ Wild type & 8 \\
Prior therapy & \\
Pleurodesis & 1 \\
$\gamma$-knife & 1 \\
EGFR-TKI (gefitinib) & 2 \\
\hline
\end{tabular}

Ad: Adenocarcinoma; EGFR: epidermal growth factor receptor.

Table II. Efficacy $(n=12)$.

\begin{tabular}{lc}
\hline & $\mathrm{n}(\%)$ \\
\hline Complete response & $0(0.0)$ \\
Partial response & $7(58)$ \\
Stable disease & $5(42)$ \\
Progressive disease & $0(0.0)$ \\
Response rate $(\%)$ & 58 \\
Disease control rate $(\%)$ & 100 \\
\hline
\end{tabular}

\section{Results}

Patient characteristics. A total of 12 patients were enrolled in this study from June 2013 to July 2017.

Despite a 1-year extension of the enrollment period, the patient accrual rate remained low and the study was terminated. Table I summarizes the baseline characteristics of the 12 patients. The median age was 80 (range $=75-83$ years) years, and 8 patients $(67 \%)$ were female. All patients had an adenocarcinoma histology, and 4 patients harbored EGFR mutations (exon 19 del, 2 patients; L858R, 2 patients). Two patients with EGFR mutation-positive disease had previously received EGFR-TKI therapy. No patients harbored an ALK translocation.

Treatment delivery. A median of 4 (range=3-4 cycles) chemotherapy cycles were administered during the induction phase. Eleven patients (92\%) were able to complete the induction phase, whereas 1 patient requested the termination

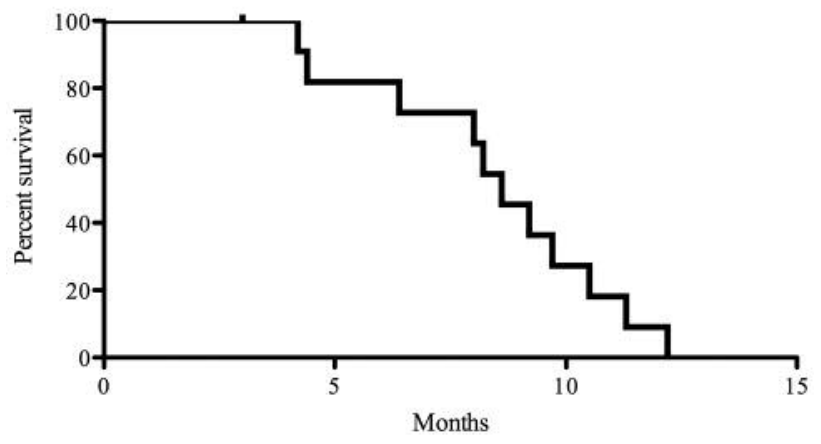

Figure 1. Kaplan-Meier analysis of progression-free survival. The median progression-free survival duration was 8.4 months (95\% confidence interval=4.4-10.5 months).

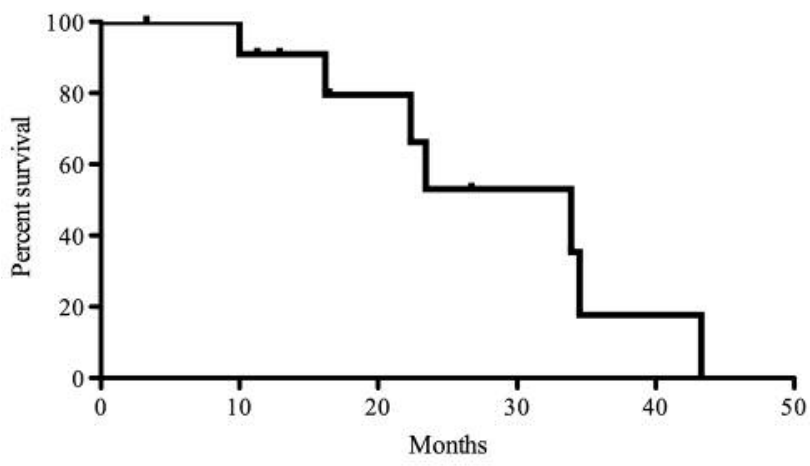

Figure 2. Kaplan-Meier analysis of overall survival. The median overall survival duration was 33.9 months (95\% confidence interval=13.2-43.3 months).

of study treatment because of AEs. Two patients required dose reductions because of non-hematologic AEs. In addition, 2 of the 11 patients were deemed ineligible for the maintenance phase because of bevacizumab-related AEs (sustained proteinuria and diverticulitis). Nine patients $(75 \%)$ initiated bevacizumab maintenance therapy and received a median of 4 (range=1-11 cycles) cycles. The following reasons were cited for the discontinuation of bevacizumab maintenance therapy: bevacizumab-related-toxicity, 3 patients; disease progression, 6 patients.

Efficacy. Among the 12 evaluable patients, none achieved a CR, 7 achieved a PR, and 5 achieved stable disease (SD). The ORR was $58 \%$, and the disease control rate (DCR; $\mathrm{CR}+\mathrm{PR}+\mathrm{SD}$ ) was $100 \%$ (Table II). Four patients remained alive at a median follow-up of 22.9 (range=6.0-43.3 months) months. The median PFS was 8.4 months [95\% confidence interval $(\mathrm{CI})=4.4-10.5$, Figure 1] and the median OS was 33.9 months (95\% CI=13.2-43.3; Figure 2). 
Safety. AEs were assessed in all 12 patients. The profiles of major hematologic and non-hematologic AEs are summarized in Table III. Grade $\geq 3$ hematologic AEs observed in this study included neutropenia (67\%), anemia $(42 \%)$, thrombocytopenia (25\%), and leukopenia (25\%). Grade 4 neutropenia was observed in 2 patients; however, no patients required granulocyte colony-stimulating factor (GCSF) administration. Grade 3 non-hematologic AEs observed in this study included febrile neutropenia (FN) (8\%), hypertension (8\%), hyponatremia (8\%), hypokalemia (17\%), elevated alanine aminotransferase levels (8\%), and diverticulitis (8\%). Two patients required carboplatin dose reductions because of AEs (alanine aminotransferase increase and $\mathrm{FN}$ ), and 5 patients discontinued the study treatment because of AEs. The following bevacizumab-related AEs were most frequently cited as reasons for discontinuation: proteinuria (17\%), colon hemorrhage (8\%) and hemosputum $(8 \%)$. Serious AEs were not observed during the study treatment.

\section{Discussion}

To our knowledge, this is the first prospective study of a combination regimen comprising carboplatin and pemetrexed plus bevacizumab followed by maintenance bevacizumab in a population of elderly patients ( $\geq 75$ years) with non-sq NSCLC. As noted above, however, the study was terminated because of slow patient accrual, despite extending the registration period. We took precautions to exclude patients considered at high risk for bevacizumab use. Therefore, the poor accrual might be attributable to the smaller than expected number of fit, elderly patients. Most patients deemed ineligible for this study had a history of cardiovascular events or required anticoagulant or antiplatelet therapy.

Elderly patients often present with factors that may affect treatment outcomes, including age-related decreases in organ function and frequent comorbidities and concomitant medication use. A retrospective analysis of the ECOG4599 study found that both grade $\geq 3$ toxicities and treatmentrelated deaths were significantly more frequent among elderly patients ( $\geq 70$ years), compared with younger patients ( $87 \% v s .70 \%, p<0.001$ and $6.3 \%$ vs. $2.6 \%$, respectively). Conversely, the AVAiL study observed a similar incidence of grade $\geq 3$ toxicity among elderly ( $\geq 65$ years) and younger patients. The toxicity profiles observed in our study were consistent with those previous reports. In addition, these toxicities were generally manageable with supportive care, and no new toxicities were observed. Approximately half of the patients in this study ultimately discontinued the study treatment because of AEs; however, most reasons cited for discontinuation involved bevacizumab-related toxicities. Although patients selected were, as much as possible, fit for bevacizumab therapy, it was difficult to predict the
Table III. Profile of major adverse events (all cycles).

\begin{tabular}{llllll}
\hline & \multicolumn{5}{c}{ Grade } \\
\cline { 2 - 6 } Adverse event & G1 & G2 & G3 & G4 & G3/4(\%) \\
\cline { 2 - 6 } Hematological & & & & & \\
Leukopenia & 3 & 5 & 3 & 0 & 25 \\
Neutropenia & 2 & 2 & 4 & 2 & 50 \\
Thrombocytopenia & 3 & 3 & 3 & 0 & 25 \\
Anemia & 2 & 3 & 5 & 0 & 42 \\
Non-hematological & & & & & \\
Febrile neutropenia & - & - & 1 & 0 & 8 \\
Fatigue & 2 & 2 & 0 & 0 & 0 \\
Anorexia & 7 & 2 & 0 & 0 & 0 \\
Nausea & 3 & 1 & 0 & 0 & 0 \\
Constipation & 5 & 0 & 0 & 0 & 0 \\
Eruption & 2 & 2 & 0 & 0 & 0 \\
AST increase & 5 & 1 & 0 & 0 & 0 \\
ALT increase & 1 & 1 & 1 & 0 & 8 \\
Alb decrease & 6 & 4 & 0 & 0 & 0 \\
Hyponatremia & 0 & 0 & 1 & 0 & 8 \\
Cre increase & 4 & 0 & 0 & 0 & 0 \\
Hypertension & 0 & 0 & 1 & 0 & 8 \\
Proteinuria & 4 & 2 & 0 & 0 & 0 \\
Epistaxis & 1 & 1 & 0 & 0 & 0 \\
Hemorrhage & 3 & 1 & 0 & 0 & 0 \\
Diverticulitis & 0 & 0 & 1 & 0 & 8 \\
\hline Pry & & & & & \\
\hline
\end{tabular}

AST: Alanine transaminase; AST: aspartate aminotransferase; Alb: albumin; Cre: creatinine.

development of bevacizumab-related toxicities to require treatment discontinuation. Further investigations are needed to optimize the selection of patients, and especially elderly patients, considered fit for chemotherapy and bevacizumab.

Although this study was limited by the small number of patients, the ORR of $58 \%$, median PFS of 8.6 months, and median OS of 33.9 months are encouraging. For comparison, a retrospective analysis of elderly patients in the ECOG4599 study yielded an ORR of 29\%, median PFS of 5.9 months, and median OS of 11.3 months (12). Furthermore, another phase II study of carboplatin, pemetrexed, and bevacizumab for elderly patients ( $\geq 70$ years) yielded an ORR of 50.9\%, median PFS of 7.0 months (95\% CI=5.9-10.1), and median OS of 13.7 months (95\% CI=9.4-16.8) (15). Our favorable survival results may be attributable to the following factors: i) 4 patients (33\%) harbored EGFR mutation, ii) $67 \%$ of patients had a PS of 0 , and iii) $91 \%$ of patients were receiving second-line or subsequent chemotherapy.

The PARAMOUNT phase III study demonstrated that the administration of maintenance pemetrexed following an induction regimen of pemetrexed plus cisplatin yielded significantly longer PFS and OS relative to placebo across all analyzed age subgroups. Furthermore, pemetrexed maintenance was well tolerated and provided patients with 
better QOL (16, 17). Consequently, the administration of maintenance pemetrexed monotherapy after pemetrexed plus cisplatin induction therapy is currently considered as standard treatment option for advanced non-sq NSCLC. However, the role of maintenance therapy remained unclear in elderly patients with NSCLC. We previously reported encouraging efficacy and safety outcomes with a combination of carboplatin plus pemetrexed in fit elderly patients ( $\geq 75$ years) with non-sq NSCLC (8). Additionally, Tamiya et al. reported similar results from a phase II study of carboplatin plus pemetrexed for elderly patients with non-sq NSCLC (9). These results support the usefulness of carboplatin plus pemetrexed for fit elderly patients ( $\geq 75$ years). Currently, a randomized phase III study to compare carboplatin plus pemetrexed followed by pemetrexed maintenance therapy with docetaxel monotherapy in fit elderly patients is ongoing in Japan, and the results of this trial may establish carboplatin plus pemetrexed as a first-line chemotherapy option for elderly patients with non-sq NSCLC.

In this study, pemetrexed for maintenance therapy was not administered because no previous study had directly investigated the effects of carboplatin plus pemetrexed followed by maintenance pemetrexed on OS. Furthermore, the efficacy and safety of a combination of pemetrexed and bevacizumab for maintenance therapy remained unclear when our study was initiated. Therefore, bevacizumab monotherapy for maintenance was administered in this study. In a recent meta-analysis, Zhang et al. found that single-agent maintenance therapy significantly improved OS relative to placebo in elderly patients, whereas a combination maintenance therapy regimen yielded no survival benefits when compared with single agent maintenance (18). Although our study included a small sample size, the efficacy outcome was similar to those of previous studies $(10,15)$. Therefore, we consider carboplatin and pemetrexed plus bevacizumab followed by bevacizumab maintenance to be a favorable candidate for the further development of treatments for elderly patients ( $\geq 75$ years).

In conclusion, carboplatin and pemetrexed plus bevacizumab followed by maintenance bevacizumab was well tolerated by elderly patients ( $\geq 75$ years) and yielded encouraging results in terms of efficacy. Although the study was limited by a small sample size, our data provided important information to support an investigation of optimal chemotherapies for elderly patients with non-sq NSCLC.

\section{Conflicts of Interest}

Kazuhiko Yamada has received honoraria from Pfizer Japan, Chugai Pharmaceutical, Eli Lilly Japan, AstraZeneca, Ono Pharmaceutical, Bristol-Myers Squibb, and MSD. Koichi Azuma has received honoraria and research funding from AstraZeneca, Boehringer Ingelheim, Ono Pharmaceutical, MSD Oncology, Bristol-Myers Squibb, and Chugai Pharma. Takaaki Tokito has received honoraria from Chugai Pharmaceutical. The other Authors declare that they have no potential conflicts of interest.

\section{Acknowledgements}

The Authors would like to thank the patients, their families, and medical staff who participated in this study. In addition, the Authors would like to thank Sintaro Kanda and Takayasu Kurata for their assistance as the safety Monitoring Committee.

\section{References}

1 Cojean I and Le Chevalier T: Chemotherapy of stage IIIB and IV non-small-cell lung cancer. Ann Oncol 6: S41-44, 1995.

2 Cancer registry and Statistics. Cancer Information Service, National Cancer Center, Japan. http://ganjohojp/ reg_stat/ statistics/dl/idexhtml\#incidence.

3 The Elderly Lung Cancer Vinorelbine Italian Study Group: Effects of vinorelbine on quality of life and survival of elderly patients with advanced non-small-cell lung cancer. J Natl Cancer Inst 91: 66-72, 1999.

4 Kudoh S, Takeda K, Nakagawa K, Takada M, Katakami N, Matsui K, Shinkai T, Sawa T, Goto I, Semba H, Seto T, Ando M, Satoh T, Yoshimura N, Negoro S and Fukuoka M: Phase III study of docetaxel compared with vinorelbine in elderly patients with advanced non-small-cell lung cancer: results of the West Japan Thoracic Oncology Group Trial (WJTOG 9904). J Clin Oncol 24: 3657-3663, 2006.

5 Langer CJ, Manola J, Bernardo P, Kugler JW, Bonomi P, Cella D and Johnson DH: Cisplatin-based therapy for elderly patients with advanced non-small-cell lung cancer: implications of Eastern Cooperative Oncology Group 5592, a randomized trial. J Natl Cancer Inst 94: 173-181, 2002.

6 Goto K, Nishiwaki Y, Saijo N, Takeda K, Katakami N, Kudoh S, Ichinose Y, Ohashi Y, Ariyoshi Y and Fukuoka M: The FourArm Cooperative Study (FACS) for advanced non-small cell lung cancer (NSCLC); a subgroup analysis in elderly patients(pts). Proc Am Soc Clin Oncol 24: Abstr 7115, 2006.

7 Quoix E, Zalcman G, Oster JP, Westeel V, Pichon E, Lavole A, Dauba J, Debieuvre D, Souquet PJ, Bigay-Game L, Dansin E, Poudenx M, Molinier O, Vaylet F, Moro-Sibilot D, Herman D, Bennouna J, Tredaniel J, Ducolone A, Lebitasy MP, Baudrin L, Laporte S, Milleron B and Intergroupe Francophone de Cancerologie T: Carboplatin and weekly paclitaxel doublet chemotherapy compared with monotherapy in elderly patients with advanced non-small-cell lung cancer: IFCT-0501 randomised, phase 3 trial. Lancet 378: 1079-1088, 2011.

8 Takeoka H, Yamada K, Azuma K, Zaizen Y, Yamashita F, Yoshida T, Naito Y, Okayama Y, Miyamoto M and Hoshino T: Phase I study of carboplatin combined with pemetrexed for elderly patients with advanced non-squamous non-small cell lung cancer. Jpn J Clin Oncol 44: 472-478, 2014.

9 Tamiya A, Tamiya M, Shiroyama T, Kanazu M, Hirooka A, Tsuji T, Morishita N, Asami K, Suzuki H, Okamoto N, Okishio K, Kawaguchi T, Hirashima T, Atagi $\mathrm{S}$ and Kawase I: Dose escalation study of carboplatin-pemetrexed followed by maintenance pemetrexed for elderly patients with advanced nonsquamous non-small-cell lung cancer. Ann Oncol 24: 980-985, 2013.

10 Sandler A, Gray R, Perry MC, Brahmer J, Schiller JH, Dowlati A, Lilenbaum R and Johnson DH: Paclitaxel-carboplatin alone or with bevacizumab for non-small-cell lung cancer. N Engl J Med 355: 2542-2550, 2006. 
11 Reck M, von Pawel J, Zatloukal P, Ramlau R, Gorbounova V, Hirsh V, Leighl N, Mezger J, Archer V, Moore N and Manegold C: Phase III trial of cisplatin plus gemcitabine with either placebo or bevacizumab as first-line therapy for nonsquamous non-small-cell lung cancer: AVAil. J Clin Oncol 27: 1227-1234, 2009.

12 Ramalingam SS, Dahlberg SE, Langer CJ, Gray R, Belani CP, Brahmer JR, Sandler AB, Schiller JH, Johnson DH and Eastern Cooperative Oncology Group: Outcomes for elderly, advancedstage non-small-cell lung cancer patients treated with bevacizumab in combination with carboplatin and paclitaxel: analysis of Eastern Cooperative Oncology Group Trial 4599. J Clin Oncol 26: 60-65, 2008.

13 Leighl NB, Zatloukal P, Mezger J, Ramlau R, Moore N, Reck M, and Manegold C: Efficacy and safety of bevacizumab-based therapy in elderly patients with advanced or recurrent nonsquamous non-small cell lung cancer in the phase III BO17704 study (AVAiL). J Thorac Oncol 5: 1970-1976, 2010.

14 Simon R: Optimal two-stage designs for phase II clinical trials. Control Clin Trials 10: 1-10, 1989.

15 Patel JD, Hensing TA, Rademaker A, Hart EM, Blum MG, Milton DT and Bonomi PD: Phase II study of pemetrexed and carboplatin plus bevacizumab with maintenance pemetrexed and bevacizumab as first-line therapy for nonsquamous non-smallcell lung cancer. J Clin Oncol 27: 3284-3289, 2009.
16 Paz-Ares L, de Marinis F, Dediu M, Thomas M, Pujol JL, Bidoli P, Molinier O, Sahoo TP, Laack E, Reck M, Corral J, Melemed S, John W, Chouaki N, Zimmermann AH, Visseren-Grul C and Gridelli C: Maintenance therapy with pemetrexed plus best supportive care versus placebo plus best supportive care after induction therapy with pemetrexed plus cisplatin for advanced non-squamous non-small-cell lung cancer (PARAMOUNT): a double-blind, phase 3, randomised controlled trial. Lancet Oncol 13: 247-255, 2012.

17 Gridelli C, de Marinis F, Thomas M, Prabhash K, El Kouri C, Blackhall F, Bustin F, Pujol JL, John WJ, San Antonio B, Zimmermann A, Chouaki N, Visseren-Grul C and Paz-Ares LG: Final efficacy and safety results of pemetrexed continuation maintenance therapy in the elderly from the PARAMOUNT phase III study. J Thorac Oncol 9: 991-997, 2014.

18 Zhang L, Gao S and He J: The role of maintenance therapy in the treatment of elderly non-small-cell lung cancer patients: a meta-analysis of randomized controlled trials. Drug Des Devel Ther 11: 3435-3440, 2017.

Received April 26, 2018

Revised May 16, 2018

Accepted May 17, 2018 